\title{
A RANDOM ERGODIC THEOREM IN VON NEUMANN ALGEBRAS
}

\author{
NGHIEM DANG-NGOC
}

\begin{abstract}
E. C. Lance has recently extended the Birkhoff ergodic theorem to noncommutative dynamical systems. Using Lance's result, we extend the random ergodic theorem of H. R. Pitt, S. M. Ulam, S. Kakutani and J. von Neumann to noncommutative context.
\end{abstract}

Introduction and notations. E. C. Lance has recently proved a beautiful extension of Birkhoff ergodic theorem to von Neumann algebra context (cf. [5]). Since then, several classical almost everywhere pointwise convergence theorems have been extended to von Neumann algebras (cf. $[1,2,6])$. In this paper we use Lance's results to prove an extension of the random ergodic theorem of H. R. Pitt, S. M. Ulam and J. von Neumann (cf. [7, 9]). Our proof follows from that of S. Kakutani (cf. [4]) and Ryll-Nardzewski (cf. [8]).

We fix a pair $(\mathcal{Q}, \rho)$ where $\mathcal{Q}$ is a von Neumann algebra and $\rho$ is a faithful normal state on $\mathcal{Q}$. As in the commutative case, we call a kernel a positive linear contraction $T$ of $\mathcal{Q}$ into itself such that $T 1=1, \rho(T a)=\rho(a), \rho\left((T a)^{*} T(a)\right) \leqslant \rho\left(a^{*} a\right), \forall a \in \mathbb{Q}$. Let $\mathfrak{S}$ be the Hilbert space of the cyclic representation of $\mathscr{Q}$ associated to $\rho, \xi$ the cyclic and separating vector. If $T$ is a kernel, we associate to $T$ a canonical contraction $\tilde{T}$ on $\mathfrak{S}$ defined by $\tilde{T}(a \xi)=(T a) \xi, \forall a \in \mathbb{Q}$.

Following E. C. Lance (cf. [5]) we say that a sequence $\left(a_{n}\right)_{n \geqslant 1}$ of $\mathbb{Q}$ converges $\rho$-almost uniformly to an element $a$ of $Q$ if for every $\varepsilon>0$, there exists a projection $e$ of $\mathcal{Q}$ such that $\rho(e) \geqslant 1-\varepsilon$ and $\lim _{n}\left\|\left(a-a_{n}\right) e\right\|=0$ (when $\mathbb{Q}$ is commutative, $\mathbb{Q}=L^{\infty}(X, \mu)$, with $(X, \mu)$ a probability space, this convergence coincides, via Egorov's theorem, with the almost everywhere pointwise convergence).

Let $(X, \mu)$ be a probability space, for each $x \in X$ we associate an automorphism $\tau_{x}$ of $\mathbb{Q}$ leaving $\rho$ invariant and we assume that for every $a \in \mathbb{Q}$, the mapping $X \ni x \rightarrow \tau_{x} a \in \mathbb{Q}$ is ultrastrongly measurable. We note $(Z, \nu)=(X, \mu)^{\mathbf{N}}, \tilde{\mathbb{Q}}=$ $L^{\infty}(Z, \nu ; \mathfrak{Q}), \tilde{\mathfrak{E}}=L^{2}(Z, \nu ; \mathfrak{Q})$.

THEOREM 1. For every $a \in \mathbb{Q}$, there exists $a_{0} \in \mathbb{Q}$ such that for $\nu$-almost every $\left(x_{1}, x_{2}, \ldots\right)$ in $X^{\mathbf{N}}$ we have

$$
\frac{1}{n} \sum_{k=1}^{n} \tau_{x_{k}} \tau_{x_{k-1}} \cdots \tau_{x_{1}} a \rightarrow a_{0}
$$

$\rho$-almost uniformly as $n \rightarrow \infty$. Furthermore $a_{0}$ is $\tau_{x}$-invariant for $\mu$-almost every $x$ in $X$.

Received by the editors November 20, 1981.

AMS (MOS) subject classifications (1970). Primary 46L10; Secondary 28 A65.

Key words and phrases. von Neumann algebras, almost uniform convergence, ergodic theorem, random ergodic theorem. 0002-9939/82/0000-1082/\$02.00 
Proof. Consider the von Neumann algebra $\tilde{\mathscr{Q}}=L^{\infty}(Z, \nu ; \mathcal{Q})$ provided with the faithful normal state $\tilde{\rho}$ :

$$
\tilde{\rho}(f)=\int_{Z} \rho(f(z)) d \nu(z), \quad \forall f \in L^{\infty}(Z, \nu ; \mathcal{Q}) .
$$

Let $T$ be the mapping of $\tilde{\mathscr{Q}}$ into itself defined by

$$
T f\left(x_{1}, x_{2}, \ldots\right)=\tau_{x_{1}} f\left(x_{2}, x_{3}, \ldots\right), \quad f \in L^{\infty}(Z, \nu ; \mathbb{Q}) .
$$

We can easily verify that $T$ is a kernel of $(\tilde{\mathscr{U}}, \tilde{\rho})$.

Let $a \in \mathbb{Q}$ and $f \in \tilde{Q}$ defined by $f(z)=a, \forall z \in Z$. We can apply Lance's ergodic theorem (cf. [5]): there exists $g \in \tilde{\mathbb{Q}}$ such that $T g=g$ and

$$
\frac{1}{n} \sum_{k=1}^{n} T^{k} f \underset{n}{\rightarrow} g \quad \tilde{\rho} \text {-almost uniformly. }
$$

(Lance proved the ergodic theorem for automorphisms but the result remains valid for kernels.)

We now need some technical results to characterize $g$.

LEMmA 1. Let $g \in \tilde{Q}$ such that $T g=g$, then there exists $a_{0} \in \mathbb{Q}$ such that $g(z)=a_{0}$ for almost every $z \in Z$. Furthermore $\tau_{x} a_{0}=a_{0}$ for almost every $x$ in $X$.

Proof. (1) Let $h \in L^{\infty}(Z, \nu)$ such that $\int_{Z} h(z) d \nu(z)=0,|h| \leqslant M$ and define

$$
\varphi=\int_{Z} g\left(x_{1}, x_{2}, \ldots\right) h\left(x_{1}, x_{2}, \ldots\right) d \mu\left(x_{1}\right) d \mu\left(x_{2}\right) \cdots \in \mathbb{Q} .
$$

From the hypothesis $T g=g$ we deduce

$$
g\left(x_{1}, x_{2}, \ldots\right)=\tau_{x_{n-1}} \tau_{x_{n-2}} \cdots \tau_{x_{1}} g\left(x_{n}, x_{n+1}, \ldots\right) .
$$

Let

$$
\varphi_{n}\left(x_{1}, x_{2}, \ldots\right)=\int_{Z} g\left(x_{1}, x_{2}, \ldots\right) h\left(x_{n}, x_{n+1}, \ldots\right) d \mu\left(x_{n}\right) d \mu\left(x_{n+1}\right) \cdots
$$

By the relations (2) and (1), we have

$$
\varphi_{n}\left(x_{1}, x_{2}, \ldots\right)=\tau_{x_{n-1}} \tau_{x_{n-2}} \cdots \tau_{x_{1}} \varphi .
$$

Consider the mapping $Z \ni z \rightarrow g(z) \xi \in \mathfrak{Q}$, and for every $\varepsilon>0$, let $g_{\varepsilon}\left(x_{1}, \ldots, x_{N}\right)$ be a strongly measurable mapping of $Z$ into $\mathfrak{Q}$ depending only on a finite number of coordinates $x_{1} \cdots x_{N}$ such that

$$
\int_{Z}\left\|g(z) \xi-g_{\varepsilon}(z)\right\| d \nu(z)<\frac{\varepsilon}{M} .
$$

For any $n \geqslant N+1$ we have

$$
\begin{aligned}
\varphi_{n}\left(x_{1}, x_{2}, \ldots\right) \xi= & \int\left(g\left(x_{1}, x_{2}, \ldots\right) \xi-g_{\varepsilon}\left(x_{1}, \ldots, x_{N}\right)\right) \\
& \times h\left(x_{n}, x_{n+1}, \ldots\right) d \mu\left(x_{n}\right) d \mu\left(x_{n+1}\right) \ldots
\end{aligned}
$$


since

$$
\begin{gathered}
\int g_{\varepsilon}\left(x_{1}, x_{2}, \ldots, x_{N}\right) h\left(x_{n}, x_{n+1}, \ldots\right) d \mu\left(x_{n}\right) d \mu\left(x_{n+1}\right) \ldots \\
=g_{\varepsilon}\left(x_{1}, \ldots, x_{n}\right) \int_{Z} h(z) d \nu(z)=0 .
\end{gathered}
$$

Combining (5) and (4) we obtain

$$
\int\left\|\varphi_{n}\left(x_{1}, x_{2}, \ldots\right) \xi\right\| d \mu\left(x_{1}\right) d \mu\left(x_{2}\right) \cdots<\frac{\varepsilon}{M} \times M=\varepsilon .
$$

Therefore the relation (3) implies that $\|\varphi \xi\|<\varepsilon, \forall \varepsilon>0$. Hence $\varphi \xi=0$ and $\varphi=0$ (since $\xi$ is faithful for $\mathcal{Q}$ ). We have proved that

$$
\int g\left(x_{1}, x_{2}, \ldots\right) h\left(x_{1}, x_{2}, \ldots\right) d \mu\left(x_{1}\right) d \mu\left(x_{2}\right) \cdots=0,
$$

whenever $h \in L^{\infty}(Z, \nu)$ and $\int h(z) d \nu(z)=0$.

(2) Let $\psi \in \mathfrak{Q}, h \in L^{\infty}(Z, \nu), h_{0}=\int_{Z} h(z) d \nu(z)$, and $a_{0}=\int_{Z} g(z) d \nu(z)$. By the above results, we have

$$
\int\left(h^{*}(z)-h_{0}^{*}\right) g(z) \xi d \nu(z)=0
$$

$\left(h^{*}(z)\right.$ is the complex conjugate of $\left.h(z)\right)$ or

$$
\int h^{*}(z)\left(g(z)-a_{0}\right) \xi d \nu(z)=0 .
$$

Therefore $\int_{Z}\left\langle\left(g(z)-a_{0}\right) \xi, h(z) \psi\right\rangle d \nu(z)=0$.

As the vectors $h(\cdot) \psi, h \in L^{\infty}(Z, \nu), \psi \in \mathfrak{Q}$, are total in $\tilde{\mathfrak{Q}}=L^{2}(Z, \nu ; \mathfrak{Q})$, we have $\left(g(z)-a_{0}\right) \xi=0$, for $\nu$-almost every $z$ and since $\xi$ is faithful for $Q$, we obtain finally $g(z)=a_{0}$ for $\nu$-almost every $z$. It is clear that $\tau_{x} a_{0}=a_{0}$ for almost every $x$ in $X$.

Lemma 1 is proved. Q.E.D.

LEMMA 2. Let $f_{n} \in \tilde{\mathscr{Q}}, f \in \tilde{\mathbb{U}}$ and assume that $f_{n} \rightarrow f \tilde{\rho}$-almost uniformly. Then for almost every $z \in Z$ we have $f_{n}(z) \rightarrow f(z) \rho$-almost uniformly.

Proof. Let $\varepsilon>0$; there exists a projection $\tilde{e} \in \tilde{\mathbb{Q}}, \tilde{e}=\int^{\oplus} e(z) d \nu(z)$ such that $\rho(\tilde{e}) \geqslant 1-\varepsilon$ and $\left\|\left(f_{n}-f\right) \tilde{e}\right\| \rightarrow 0, n \rightarrow \infty$. This implies that $\left\|\left(f_{n}(z)-f(z)\right) e(z)\right\|$ $\rightarrow 0$ for almost every $z \in Z$. Let $Z_{\varepsilon}=\left\{z \in Z / \rho(e(z)) \geqslant 1-\varepsilon^{1 / 2}\right\}$; as $\tilde{\rho}(\tilde{e})=$ $\int_{Z} \rho(e(z)) d \nu(z) \geqslant 1-\varepsilon$ we must have

$$
1-\varepsilon \leqslant \tilde{\rho}(\tilde{e}) \leqslant \nu\left(Z_{\varepsilon}\right)+\left(1-\varepsilon^{1 / 2}\right)\left(1-\nu\left(Z_{\varepsilon}\right)\right), \quad \nu\left(Z_{\varepsilon}\right) \geqslant 1-\varepsilon^{1 / 2} .
$$

Now let $\varepsilon_{k}=1 / 2^{k}, \tilde{e}_{\varepsilon_{k}}, Z_{\varepsilon_{k}}$ as above and consider $Z^{\prime}=\cup_{n \geqslant 1} \cap_{k \geqslant n} Z_{\varepsilon_{k_{k}}}$. We have $\nu\left(Z^{\prime}\right)=1$, and for $z \in Z^{\prime}$ we have $f_{n}(z) \rightarrow f(z) \rho$-almost uniformly. Q.E.D.

The theorem follows from Lemmas 1 and 2.

We also have a converse of the above lemma in the case $(Z, \nu)$ standard and $\mathbb{Q}$ countably generated:

LEMMA 3. Assume that $(Z, \nu)$ standard and $\mathbb{Q}$ countably generated, let $f_{n} \in \tilde{\mathbb{Q}}, f \in \tilde{\mathbb{Q}}$. Then $f_{n}$ converges $\tilde{\rho}$-almost uniformly to $f$ if and only if $f_{n}(z)$ converges $\rho$-almost uniformly to $f(z)$ for $\nu$-almost every $z$ in $Z$. 
Proof. Assume that for almost every $z \in Z$. we have $f_{n}(z) \rightarrow f(z) \rho$-almost uniformly as $n \rightarrow \infty$.

Let $\mathcal{L}_{1}$ be the set of contractions of $\mathscr{Q}$ into itself provided with the strong operator topology, $L_{1}$ is a standard Borel space.

For every $\varepsilon>0$, let $Q$ be the set of pairs $(z, e) \in Z \times L_{1}$ such that

(1) $e$ is a projection, $e \in \mathbb{Q}$.

(2) $\langle e \xi, \xi\rangle \geqslant 1-\varepsilon / 2$.

(3) $\lim _{n \rightarrow \infty} \sup _{k>n}\left\|\left(f_{k}(z)-f(z)\right) e\right\|=0$.

The set $Q$ is a Borel subset of $Z \times \mathscr{L}_{1}$. Following [3, appendice V], there exists a Borel mapping $Z \ni z \rightarrow e(z) \in \mathcal{L}_{1}$ such that $(z, e(z)) \in Q, \nu$-a.e.

We have

$$
\lim _{n} \sup _{k \geqslant n}\left\|\left(f_{k}(z)-f(z)\right) e(z)\right\|=0 \quad \nu \text {-a.e. }
$$

By Egorov's theorem, there exists a Borel subset $Z_{\varepsilon}$ of $Z$ such that $\nu\left(Z_{\varepsilon}\right) \geqslant 1-\varepsilon / 2$ and

$$
\sup _{z \in Z_{\varepsilon}}\left\|\left(f_{n}(z)-f(z)\right) e(z)\right\| \rightarrow 0, \quad n \rightarrow \infty .
$$

Let $\tilde{e}=\int^{\oplus} e(z) 1_{z^{\prime}}(z) d \nu(z)$; we have $\tilde{\rho}(\tilde{e}) \geqslant 1-\varepsilon$ and $\left\|\left(f_{n}-f\right) \tilde{e}\right\| \rightarrow 0, n \rightarrow \infty$. Therefore $f_{n} \rightarrow_{n} f \tilde{\rho}$-almost uniformly. The lemma is proved.

REMARKS. The proofs of Lemmas 2 and 3 remain valid for nonconstant fields of von Neumann algebras.

\section{REFERENCES}

1. J. P. Conze and N. Dang-Ngoc, Ergodic theorems for non commutative dynamical systems, Invent. Math. 46 (1978), 1-15.

2. N. Dang-Ngoc, Pointwise convergence of martingales in von Neumann algebras, Israel J. Math. 34 (1979), 273-280.

3. J. Dixmier, Les algèbres d'opérateurs dans l'espace hilbertien, Gauthier-Villars, Paris, 1969.

4. S. Kakutani, Random ergodic theorems and Markoff processes with a stable distribution, (Proc. Second Berkeley Sympos. on Mathematical Statistics and Probability, 1950), Univ. of California Press, Berkeley and Los Angeles, 1951, pp. 247-261.

5. E. C. Lance, Ergodic theorem for convex sets and operator algebras, Invent. Math. 37 (1976), 201-211.

6. _ Martingale convergence in von Neumann algebras, Math. Proc. Cambridge Philos. Soc. 84 (1978).

7. H. R. Pitt, Some generalizations of the ergodic theorem, Math. Proc. Cambridge Philos. Soc. 38 (1952), 325-343.

8. C. Ryll-Nardzewski, On the ergodic theorems. III. The random ergodic theorem, Studia Math. 14 (1954), 298-301.

9. S. M. Ulam and J. von Neumann, Random ergodic theorem, Bull. Amer. Math. Soc. 51 (1954), 660.

Departement de Mathematiques, Université de l'Etat a Mons, 7000 Mons, Belgique

Laboratoire de Probabilites, Tour 56, Universite de Pierre et Marie Curie, 75230 Paris Cedex 05, FraNCE 